\title{
Totally Accessible MRI: A User's Guide to Principles, Technology, and Applications
}

\author{
M.L. Lipton
}

New York, NY: Springer, 2008, 313 pages, \$59.95

This book was written for an audience of clinical radiologists, nuclear medicine physicians, residents in radiology and nuclear medicine, technologists, medical health physicists, and any individual interested in understanding MRI as used in clinical practice today. The purpose of the book, as explained by the author, is to help the reader develop a clear understanding of MRI, including essential principles for facilitating and enhancing the application of clinically relevant imaging techniques. The content is presented as 19 chapters and 4 supporting appendices. The chapters are divided into 3 distinctive parts that help the reader understand the nuclear magnetic resonance signal as a concept unrelated to imaging, basic imaging components, and advanced MRI applications.

The content of this book was developed from a course on the physical basis and practical use of MRI technology offered at the undergraduate and graduate levels but primarily attended by clinical radiologists and residents. The reader is encouraged to learn the fundamentals of MRI technology as presented in a step-by-step format that explains the single common goal of MRI technology: "to differentiate tissue in 2 adjacent locations based on the way that tissue behaves in the MRI environment." Part I begins the learning process quite literally by starting from the ground up. Topics of discussion include image contrast, which is central to clinical images; nuclear magnetism; nuclear spin; and nuclear spin angular momentum. The discussion relies heavily on quantum mechanics and can be daunting to understand outside the classroom environment. The groundwork continues with a detailed discussion of transverse magnetization and radiofrequency as they relate to excitation and relaxation. Spin-echo and longitudinal and transverse relaxation times (T1, T2, and $\mathrm{T} 2 *$ ) as they pertain to image contrast and photon density are discussed and remain core concepts in generating, measuring, and manipulating the MRI signal. The final chapter in part I reviews gradient magnetic fields in terms of current hardware- that is, the types of magnets available today in MRI technology, as well as radiofrequency coils, shielding, and computers. Part II moves the discussion from producing the MRI signal to locating and measuring the signal in tissue and differentiating the signal coming from different types of tissue (e.g., normal vs. pathologic). Key terms familiar in

COPYRIGHT (C) 2008 by the Society of Nuclear Medicine, Inc. DOI: $10.2967 /$ jnmt.108.057539 nuclear medicine, including slice thickness, slice localization, spatial localization, and spatial resolution, are presented as they relate to MRI. Throughout this discussion, the reader is continually reminded that in MRI technology, frequency is location. The complexity of the discussion regarding single and two-dimensional Fourier transformation easily makes it one of the most difficult topics to understand in the book. The remainder of part II enters the more practical world of MRI, including variables that affect field of view, as well as pulse sequences; multiple echoes, multiple slices, and gradient echo. Quality assurance, quality control, and safety are critical to any imaging modality. The author presents an excellent and detailed discussion on the variables in MRI technology affecting both image quality and safety. Part III provides what the author admits is a somewhat superficial discussion of advanced MRI techniques, including saturation techniques, fast imaging, volumetric imaging, parallel imaging, angiography, diffusion detection, magnetic susceptibility, spectroscopy, and spectroscopic imaging. Although the author believes, as do I, that each of these topics warrants an entire book for adequate discussion, to the beginner in MRI technology each chapter provides a basic understanding of the subject that can be enhanced, as desired, with further reading.

The author assumes the reader to have no background knowledge of MRI technology other than the most basic of concepts on vectors, including their addition and decomposition (presented in appendix 1). However, although the writing is in a nonmathematic format, the complexity associated with the creation of the nuclear magnetic resonance signal (part 1) and the basics of MRI (part II) may at times make the novice wonder if prerequisite courses in quantum physics and associated topics might facilitate understanding. Additionally, without the support of appendix 2 (glossary of terms) and appendix 3 (common MRI acronyms, abbreviations, and notations), apprentices in MRI technology may find themselves hopelessly lost in the many terms and concepts important to the discussion. Although this topic is presented in what the author believes is a "user-friendly format," from my viewpoint the book could have a significantly increased value if used in a classroom format to allow further discussion of the more complex topics.

Appendix 4 serves as a valuable resource for those looking for additional references for further study.

Kathy S. Thomas Battle Ground, Washington 